\title{
Image Processing using Principle Component Analysis
}

\author{
Pramod Kumar Pandey \\ Department of Electrical and \\ Instrumentation Engineering \\ Thapar University, Patiala \\ Punjab, India
}

\author{
Yaduvir Singh \\ Department of Electrical and \\ Instrumentation Engineering \\ Thapar University, Patiala \\ Punjab, India
}

\author{
Sweta Tripathi \\ Mewar university Chittorgarh \\ Rajasthan-321901, India
}

\begin{abstract}
In this paper, a review on the latest methodologies and application of the Principle Component Analysis (PCA) has been done in the area of image processing. Exploring basic theory of multivariate analysis, which involves a mathematical procedure to transform a number of correlated variables into a number of uncorrelated variables have been studied, compared and analyzed for better performance. The PCA ultimately reduces the number of effective variables used for classification which are compared with some statistical method. A comparison is made to illustrate the important of PCA in various signal processing based application like Texture classification, Face recognition, Biometrics etc.
\end{abstract}

Keywords: Principle Component Analysis, Maltivariate Analysis

\section{INTRODUCTION}

Image Processing Toolbox provides a comprehensive set of reference-standard algorithms and graphical tools for image processing, analysis, visualization, and algorithm development. We can restore noisy or degraded images, enhance images for improved intelligibility, extract features, analyze shapes and textures, and register two images. Most toolbox functions are written in the open MATLAB ${ }^{\circledR}$ language, giving we the ability to inspect the algorithms, modify the source code, and create our own custom functions.

Image Processing Toolbox supports engineers and scientists in areas such as biometrics, remote sensing, surveillance, gene expression, microscopy, semiconductor testing, image sensor design, color science, and materials science. It also facilitates the learning and teaching of image processing techniques.

The Principal Component Analysis (PCA) is one of the most successful techniques that have been used in image recognition and compression. PCA is a statistical method under the broad title of factor analysis. The purpose of PCA is to reduce the large dimensionality of the data space (observed variables) to the smaller intrinsic dimensionality of feature space (independent variables), which are needed to describe the data economically. This is the case when there is a strong correlation between observed variables.

The jobs which PCA can do are prediction, redundancy removal, feature extraction, data compression, etc. Because PCA is a classical technique which can do something in the linear domain, applications having linear models are suitable, such as signal processing, image processing, system and control theory, communications, etc.

Face recognition has many applicable areas. Moreover, it can be categorized into face identification, face classification, or sex determination. The most useful applications contain crowd surveillance, video content indexing, personal identification (ex. driver's license), mug shots matching, entrance security, etc. The main idea of using PCA for face recognition is to express the large 1-D vector of pixels constructed from 2-D facial image into the compact principal components of the feature space. This can be called eigen space projection. Eigen space is calculated by identifying the eigenvectors of the covariance matrix derived from a set of facial images (vectors).

In this paper, a review on the latest methodologies and applications of the Principal Component Analysis (PCA) has been done in the area of Image processing. Exploring basic theory of multivariate analysis, which involves a mathematical procedure to transform a number of correlated variables into a number of uncorrelated variables some major applications have been studied.

\section{LITERATURE REVIEW OF RECENT PCA WORKS}

\subsection{Two Dimensional PCA for Face Recognition}

It is an image projection technique developed for image feature extraction. As compared to PCA, it is based on 2D matrices rather than $1 \mathrm{D}$ vector so the image matrix does not need to be transformed into a vector prior to feature extraction [2]. Instead, an image covariance matrix is constructed directly using the original image matrices. Also the size of the image covariance matrix is much smaller in 2DPCA as compared to that of PCA. Therefore, 2DPCA has two important advantages over PCA. First, it is easier to evaluate the covariance matrix accurately. Second, less time is required to determine the corresponding eigenvectors [3].

\subsection{Face Verification}

The human face is a smart source of biometric information, from which unfair measurements can be acquired naturally without much user interaction relative to other biometric information. The recognition of faces has been an entrenched field of research, with a large number of algorithms proposed in the past thirty years. Though it is an easy task for human beings to recognize a person from just one or several photos, this is not the case for computers. Automatic face recognition has been recognized as one the most challenging tasks in pattern recognition and artificial intelligence [7].

Verification of identity based on biometric information is essential for many security applications, since the conventional authentication approaches, e.g. user/password mechanisms, have proved unreliable and inconvenient. Examples include access control to physical facilities, security systems or information databases. Suspect tracking, Surveillance and intrusion detection are also potential applications. In addition to the wide range of commercial and law enforcement applications mentioned above, there are many emerging fields that can benefit from face verification technology, such as the new generation of intelligent humancomputer interfaces and e-services, including teleshopping and tele-banking. There exist many optional biometric cues for identity verification, such as the iris, fingerprint, voice, 
handprint, signature, and retina. The human face plays an exceptional role in biometrics technology due to some of its unique characteristics. First, most cameras are non-invasive; therefore face verification systems are one of the most publicly acceptable verification technologies in use. Another advantage is that face recognition systems can work mostly without the cooperation of the user concerned, which is therefore very convenient for the general users. Furthermore, they can even work in the situation where the subject concerned is not aware of the procedure. This point greatly facilitates applications such as criminal hunting, surveillance, tracking shoplifters, suspect tracking and investigation, etc. The machine recognition of faces can be formulated as follows: given still or video images of a scene, identify one or more persons in the scene using a stored database of faces.

There are following 2 types of applications in Face Recognition:

1) Face recognition/Identification: In this, the input to the system is face image and the system reports the decided identity of the person on the basis of database of known individuals.

2) Face Verification: In this, the system confirms or rejects the claimed identity according to the input face image.

\subsection{Automatic Location of Optic Disc in Retinal Images}

The diagnosis and treatment of various eye diseases use the photographs of the retinal fundus. It is also one of the main resources for mass screening of diabetic retinopathy. The ophthalmologists can increase the productivity and efficiency in clinical environment if they are able to quickly process a large number of fundus images. The optic disk is the brightest part in the normal fundus image that can be seen as a pale, round or vertically slightly oval disk. It is the entrance region of blood vessels and optic nerves to the retina and its detection is essential since it often works as a reference for the other features in the fundus image [1].

There are two strategies of retinal fundus image analysis. One is bottom-up processing which is applied to the detection of optic disk by many research groups. In this approach the optic disk is identified by the largest area of pixels having highest gray level in the images. The method works well in normal fundus images. While processing the images with a large area of exudates, it will give the wrong location since the color and intensity of exudates are 105 similar to optic disk. Another strategy called top-down processing is also implemented in this paper as a model based method to locate the optic disk automatically [8]. The proposed method combines the two strategies. First, the candidate regions are determined by the bottom-up processing. Then the PCA (Principal Component Analyses) based model approach is applied to the candidate regions to give the final location of optic disk. The candidate regions are defined by clustering the brightest pixels in retinal image. PCA method with different scaling factors is applied to the candidate regions to find the minimum distance between the original input image and its projection onto the "disk space". The center of optic disk is located at the point with the minimum distance in the candidate regions among all the scaling factors. The approximate size of optic disk can also be obtained during the processing. The comparison between the proposed method and other method was also performed. The experimental results demonstrate that the proposed algorithm is more robust especially with the presence of large area of light lesions [5].

\subsection{Texture Analysis}

Textures are characteristic intensity variations that typically originate from roughness of object surfaces. For a well defined texture, intensity variations will normally exhibit both regularity and randomness and for this reason texture analysis requires careful design of statistical measures [13].

In traditional image processing literature, there are primarily three different approaches used to describe the texture of a region in an image. The three approaches are statistical, structural and spectral texture analysis. Statistical texture analysis techniques primarily describe texture of regions in an image through moments of its grayscale histogram. According to the number of pixels defining the local region (i.e. feature), statistics can be divided into first, second, or higher moments of the grayscale histogram [13]. Using statistical analysis one can characterize textures as smooth, coarse, grainy etc. On the other hand, structural texture analysis techniques decompose a pattern in an image into texture elements. In structural analysis the properties and placement rules of the texture elements define the texture. Finally, spectral texture analysis techniques are based on the properties of the Fourier spectrum of an image. Fourier transforms detect global periodicity in images, producing high-energy peaks in their spectrum at those wavelengths that describe the major periodic components in the pixel brightness throughout the image [13].

Image data when collected in multiple variables forms a three-dimensional data set where the third dimension represents the variables. Such multivariate image data consists of a stack of congruent images, where each pixel location in the image plane is represented by multiple brightness values. As a result, there is an enormous amount of highly correlated data in multivariate images. This is accomplished by compressing the highly correlated data into a reduced dimensional subspace through a few linear combinations of the brightness values per pixel location in the image plane. A 3 -way data matrix $X$ can generally represent the threedimensional pixel intensity data of a multivariate image. Upon performing a multi-way PCA decomposition on X one can represent the multivariate image in a subspace, called the Latent Variable (LV) space of MIA [9].

\section{PROBLEM FORMULATION}

In traditional PCA the larger eigenvectors corresponding with the larger eigenvalues are selected as principal components, which make the variance of data minimum. However, there are still some questions of PCA should be addressed as the following. Firstly, The idea of PCA is correct from image compression point of view; keeping the largest nonzero principal components means that we keep most of the energy (information) of that image by projecting into lower dimension subspace. However, from the pattern classification point of view, this argument may not be true. The main reason is that, in pattern classification we would like to find a set of projection vectors that can provide the highest discrimination between different classes. Thus, choosing the largest principal components as the bases for dimensionality reduction may not be optimal. Secondly, PCA is unsupervised and statistical type algorithm to extract features in input data since it does not use the class information of input data. In this case, the extracted features may not be fitted for classifications. Therefore, the principal components are not always useful for classification since they are not the most discriminating feature. Some of the small principal components may have better performance of classification than the selected large principal components. 
In other words, a strategy for selecting some smaller principal components is required.

\subsection{Local PCA Algorithms}

A number of algorithms have been proposed to extract the first $\mathrm{p}$ principal components from $\mathrm{d}$ dimensional $(\mathrm{d}>\mathrm{p})$ stochastic process with the help of a neural network. These algorithms are advantageous in adapting online to the data and thus no explicit computation of the covariance matrix and its eigenvalue decomposition is necessary. In local Hebbian type learning algorithms, the modification of the Ith row of the Weight matrix $\mathrm{W}$ between input and output layer depends only on the Ith output unit and the input. Due to this locality it has been argued that these algorithms are "biologically plausible". In such local algorithms have been analyzed and it was shown that only one part of these algorithms, the asymmetric algorithms, where there is a hierarchical connection between the output units, can lead to asymptotically stable equilibrium, whereas algorithms with a symmetric connection in the output layer is not asymptotically stable. Thus, it was concluded that asymmetric algorithms should be preferred, although they disallow "competition" and lack symmetry.

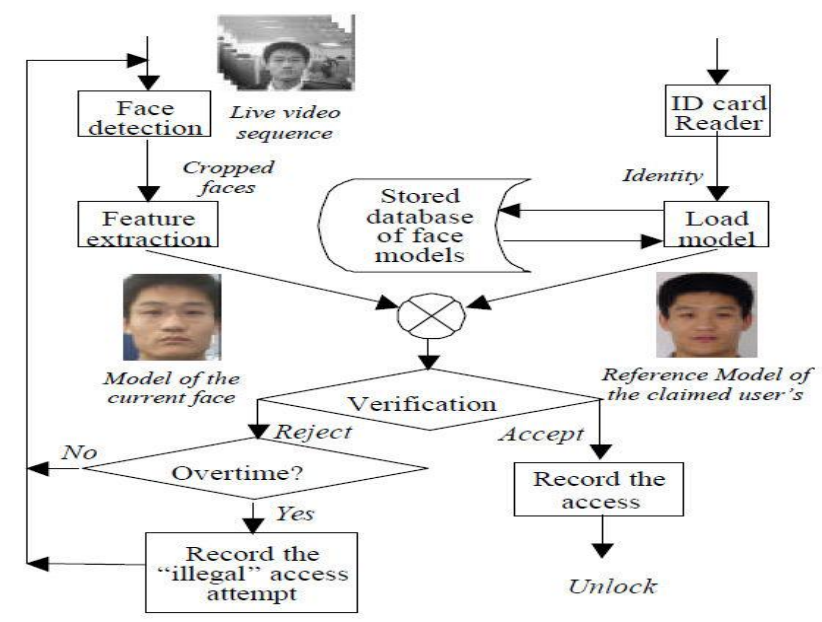

Figure 1: PCA process

\subsection{PCA Mathematical Modelling}

A 2-D facial image can be represented as $1-D$ vector by concatenating each row (or column) into a long thin vector. Let's suppose we have $\mathrm{M}$ vectors of size $\mathrm{N}$ (= rows of image $£$ columns of image) representing a set of sampled images. pj's represent the pixel values.

$$
\mathrm{xi}=\left[\mathrm{p}_{1} \cdots \cdots \cdots \cdot \mathrm{p}_{\mathrm{n}}\right]^{\mathrm{T}}, i=1 \cdots \cdots \cdots \cdot \mathrm{M}
$$

The images are mean centered by subtracting the mean image from each image vector. Let $\mathrm{m}$ represent the mean image.

$$
\mathrm{m}=\frac{1}{M} \sum_{k=1}^{M} \mathrm{X}_{\mathrm{i}}
$$

And let $w i$ be defined as mean centered image

$$
w i=x i \square m
$$

Our goal is to find a set of ei's which have the largest possible projection onto each of the wi's.
We wish to find a set of $\mathrm{M}$ orthonormal vectors ei for which the quantity is maximized with the orthonormality constraint

$$
\begin{gathered}
\lambda_{i}=\frac{1}{M}+\sum_{n=1}^{M}\left(\mathrm{e}_{\mathrm{i}}^{\mathrm{T}} w_{n}\right)^{2} \\
e_{l}^{T} e_{k}=\delta_{l k}
\end{gathered}
$$

It has been shown that the ei's and ,i's are given by the eigenvectors and eigenvalues of the covariance matrix

$$
C=W W^{T}
$$

Where $\mathrm{W}$ is a matrix composed of the column vectors wi placed side by side. The size of $\mathrm{C}$ is $\mathrm{N} \times \mathrm{N}$ which could be enormous. For example, images of size $64 \times 64$ create the covariance matrix of size $4096 \times 4096$. It is not practical to solve for the eigenvectors of $\mathrm{C}$ directly. A common theorem in linear algebra states that the vectors ei and scalars $K$ i can be obtained by solving for the eigenvectors and eigenvalues of the $\mathrm{M} £ \mathrm{M}$ matrix WTW .

Let di and $\mu \mathrm{i}$ be the eigenvectors and eigenvalues of WTW respectively.

$$
W^{T} W . d i=\mu_{\mathrm{i}} d i
$$

By multiplying left to both sides by $W$

$$
W W^{T}(W d i)=\mu_{\mathrm{i}}(W d i)
$$

Which means that the first $\mathrm{M} \square 1$ eigenvectors ei and eigenvalues $K \mathrm{i}$ of WWT are given by Wdi and $\mu \mathrm{i}$ respectively. Wdi needs to be normalized in order to be equal to ei. Since we only sum up a finite number of image vectors, $\mathrm{M}$, the rank of the covariance matrix cannot exceed $\mathrm{M} \square 1$ (The $\square 1$ come from the subtraction of the mean vector $m$ ).

The eigenvectors corresponding to nonzero eigenvalues of the covariance matrix produce an orthonormal basis for the subspace within which most image data can be represented with a small amount of error. The eigenvectors are sorted from high to low according to their corresponding eigenvalues. The eigenvector associated with the largest eigenvalue is one that reflects the greatest variance in the image. That is, the smallest eigenvalue is associated with the eigenvector that finds the least variance. They decrease in exponential fashion, meaning that the roughly $90 \%$ of the total variance is contained in the first $5 \%$ to $10 \%$ of the dimensions.

A facial image can be projected onto $\mathrm{M}^{\prime \prime}(\ll \mathrm{M})$ dimensions by computing

$$
\Omega=\left[\mathrm{v} 1 \mathrm{v} 2 \ldots \ldots \ldots \ldots \mathrm{v}_{\mathrm{M}^{\prime}}\right]^{\mathrm{T}}
$$

Where vi = eTiwi, vi is the Ith coordinate of the facial image in the new space, which came to be the principal component.

The vectors ei are also images, so called, eigenimages, or eigenfaces in our case. They can be viewed as images and indeed look like faces. So $\Omega$ describes the contribution of each eigenfaces in representing the facial image by treating the eigenfaces as a basis set for facial images. The simplest method for determining which face class provides the best 
description of an input facial image is to find the face class $\mathrm{k}$ that minimizes the Euclidean distance

$$
\mathrm{Ek}=\|((\Omega-\Omega \mathrm{K}))\|
$$

Where $\Omega \mathrm{K}$ is a vector describing the kTh face class? If Ek is less than some predefined threshold $\theta \mathrm{K}$ a face is classified as belonging to the class $\mathrm{k}$.

\section{RESULTS AND DISCUSSIONS}

The 2DPCA method has been used for face recognition and tested on three well-known face image databases (ORL, AR, and Yale). The ORL database has been used to evaluate the performance of 2DPCA under conditions where the pose and sample size are varied. The AR database has been employed to test the performance of the system under conditions where there is a variation over time, in facial expressions, and in lighting conditions. The Yale database has been used to examine the system performance when both facial expressions and illumination are varied. In all the 3 experiments, the recognition rate of 2DPCA was found to be superior to PCA. The recognition accuracy of 2DPCA is better than PCA in all experiments. Also, 2DPCA can improve the speed of image feature extraction significantly. But, 2DPCA based image representation is not as efficient as PCA in terms of storage requirements because 2DPCA requires more coefficients than PCA. 2DPCA performs better than PCA in face recognition because 2DPCA is more suitable for small sample size problems (like face recognition) since its image covariance matrix is quite small. Image representation and recognition based on PCA (or 2DPCA) is statistically dependent on the evaluation of the covariance matrix (although for PCA the explicit construction of the covariance matrix can be avoided). The obvious advantage of 2DPCA over PCA is that the former evaluates the covariance matrix more accurately
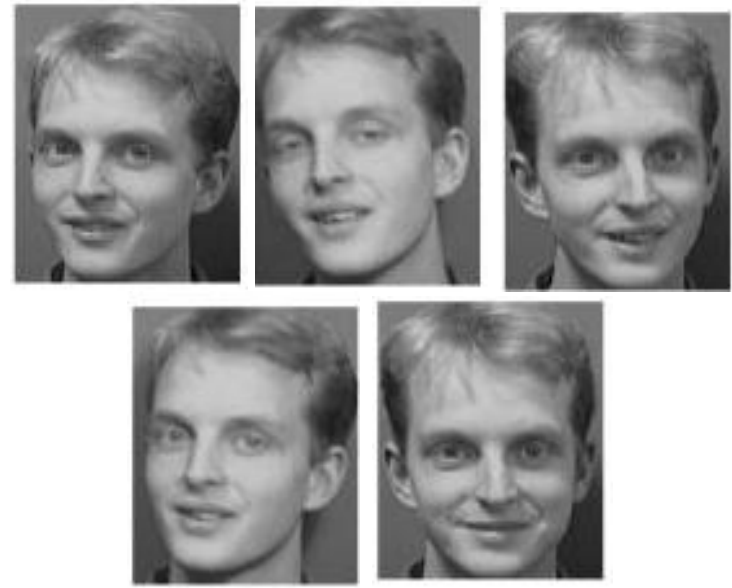

Fig 2: Five sample images of one object in the ORL face database

Work out the covariance between the $\mathrm{X}$ and $\mathrm{Y}$ dimensions in the following 2 dimensional data set, and describe what the result indicates about the data.

\begin{tabular}{|c|l|l|l|l|l|}
\hline $\begin{array}{l}\text { Item } \\
\text { Number }\end{array}$ & 1 & 2 & 3 & 4 & 5 \\
\hline $\mathrm{X}$ & 10 & 39 & 19 & 23 & 28 \\
\hline $\mathrm{Y}$ & 43 & 13 & 32 & 21 & 20 \\
\hline
\end{tabular}

\section{CONCLUSIONS}

We can conclude that Principal component analysis has been used in a number of fields. Case studies show that using for multivariate analysis, PCA analysis performs much better than in reducing the number of independent variables than those by Statistical analysis.

In future, some of small principle component may have better performance of classification than the selected large principle components. So we should add some smaller principle component while removing some large principle component i.e. we select the optimal principle component algorithm.

\section{REFERENCES}

[1] Huiqi Li, Opus Chutatape, "Automatic Location of optic Disc in retinal Images," IEEE Explore, pp. 837- 840, Oct 2008

[2] Myoung Soo Park and Jin Hee Na et al, "pca-based feature extraction using class information", IEEE International Conference on Systems, Man and Cybernetics, Vol.1, Oct. 2005, pp.341-345.

.[3] J. Yang, D. Zhang, Alejandro F. Firangi, Jing-yu Yang, "Two-Dimensional PCA: A New Approach to appearance-Based Face Representation and Recognition," IEEE transactions on pattern analysis and machine intelligence, vol. 26, no. 1, pp. 131-137, 2004

[4] A. Weingessel and K. Hornik, "Local PCA Algorithms," IEEE transactions on neural networks, vol.11, no. 6, pp 1242-1250, Nov 2000.

[5] Alex Pentland, Baback Moghaddam, and Thad Starner, "View-Based and Modular Eigen spaces for Face Recognition", IEEE Conf. on Computer Vision and Pattern Recognition, MIT Media Laboratory Tech. Report No. 2451994.

[6] K.Hornik and C.M. Kuan, "Convergence Analysis of Local feature Extraction Algorithms," Neural Networks, vol. 5, pp. 229-240, 1992.

[7] M. Turk and A. Pent land, "Eigenfaces for Recognition," J. Cognitive Neuroscience, vol. 3, no. 1, pp. 71-86, 1991.

[8] M.A. Turk and A.P. Pent land, "Face Recognition Using Eigenfaces", IEEE Conf. on Computer Vision and Pattern Recognition, pp. 586-591, 1991.

[9] F. Tomita, S. Tsuji, "Computer Analysis of Visual Textures," Kulwer Academic, Boston, 1990.

[10 ] M. Kirby and L. Sirovich, "Application of the KL Procedure for the Characterization of Human Faces," IEEE Trans. Pattern Analysis and Machine Intelligence, vol. 12, no. 1, pp. 103-108, Jan. 1990

[11] Wen Gao and Shiguang Shan, "Face Verification for Access Control," chapter 13, pp. 138.

[12] L. Sirovich and M. Kirby, "Low-Dimensional Procedure for Characterization of Human Faces," J. Optical Soc. Am., vol. 4, pp. 519-524, 1987.

[13] M.H. Bharati, John F. MacGregor, "Texture analysis of images using Principal Component Analysis," McMaster University, Hamilton, Ont., Canada, L8S 4L7. 